\title{
BM Global Heath Implementing the WHO Safe Childbirth Checklist: lessons from a global collaboration
}

\author{
WRG Perry, ${ }^{1}$ S Bagheri Nejad, ${ }^{1} \mathrm{~K}$ Tuomisto, ${ }^{1} \mathrm{~N}$ Kara, ${ }^{2} \mathrm{~N}$ Roos, ${ }^{3}$ TR Dilip, ${ }^{3}$ \\ LR Hirschhorn, ${ }^{4}$ I Larizgoitia, ${ }^{5}$ K Semrau, ${ }^{2,6,7}$ M Mathai, ${ }^{3,8}$ N Dhingra-Kumar ${ }^{1}$
}

To cite: Perry WRG, Bagheri Nejad S, Tuomisto K, et al. Implementing the WHO Safe Childbirth Checklist: lessons from a global collaboration. BMJ Glob Health 2017;2:e000241. doi:10.1136/ bmjgh-2016-000241

Received 13 November 2016 Accepted 18 April 2017

\section{CrossMark}

${ }^{1}$ Department of Service Delivery and Safety, World Health Organization, Geneva, Switzerland

${ }^{2}$ Ariadne Labs at Brigham and Women's Hospital and the Harvard T H Chan School of Public Health, Boston, Massachusetts, USA ${ }^{3}$ Department of Maternal, Newborn, Child and Adolescent Health, World Health Organization, Geneva, Switzerland

${ }^{4}$ Department of Medical

Social Sciences, Northwestern University Feinberg School of Medicine, Chicago, Illinois, USA ${ }^{5}$ Evaluation Office, World Health Organization, Geneva, Switzerland

${ }^{6}$ Department of Medicine, Harvard Medical School, Boston Massachusetts, USA

${ }^{7}$ Division of Global Health Equity, Brigham and Women's Hospital, Boston, Massachusetts, USA ${ }^{8}$ Centre for Maternal and Newborn Health, Department of International Public Health, Liverpool School of Tropical Medicine, Liverpool, UK

Correspondence to Dr S Bagheri Nejad; bagherinejads@who.int

\section{ABSTRACT}

The WHO Safe Childbirth Checklist (SCC) was developed to ensure the delivery of essential maternal and perinata care practices around the time of childbirth. A research collaboration was subsequently established to explore factors that influence use of the Checklist in a range of settings around the world. This analysis article presents an overview of the WHO SCC Collaboration and the lessons garnered from implementing the Checklist across a diverse range of settings. Project leads from each collaboration site were asked to distribute two surveys. The first was given to end users, and the second to implementation teams to describe their respective experiences using the Checklist. A total of 134 end users and 38 implementation teams responded to the surveys, from 19 countries across all levels of income. End users were willing to adopt the SCC and found it easy to use. Training and the provision of supervision while using the Checklist, alongside leadership engagement and local ownership, were important factors which helped facilitate initial implementation and successful uptake of the Checklist. Teams identified several challenges, but more importantly successfully implemented the WHO SCC. A critical step in all settings was the adaptation of the Checklist to reflect local context and national protocols and standards. These findings were invaluable in developing the final version of the WHO SCC and its associated implementation guide. Our experience will provide useful insights for any institution wishing to implement the Checklist.

\section{INTRODUCTION}

Despite significant progress in the delivery of maternal and child healthcare in recent years, there is still a high burden of mortality and morbidity among women of reproductive age and in the newborn. ${ }^{1-3}$ This is particularly so in low and middle-income countries. In 2015, it was estimated that 303000 women died around the time of pregnancy and childbirth. $^{3} \quad$ Furthermore, approximately 2.7 million newborns died within the first month of life which represents $45 \%$ of all deaths in those under the age of $5 .{ }^{1}$ There were also 2.6 million stillbirths, half of which were estimated to have occurred during labour. $^{2}$ Many of these deaths could have been prevented. ${ }^{1-4}$

Much of the early work in this field focused on improving access, such as facility-based deliveries by skilled birth attendants. This however resulted in a higher proportion of preventable deaths occurring at the health facilities. ${ }^{2}{ }^{4}$ Subsequently, a WHO-led multicountry study suggested that the provision of life-saving interventions alone is not enough to reduce maternal mortality, but that provision needs to be matched with improvements in the quality of the care. ${ }^{5}$ Indeed, poor quality of care has been identified as the single major barrier to improving mortality and morbidity around the time of birth. ${ }^{56}$

To address this challenge, WHO has embarked on several initiatives, such as Every Newborn Action Plan ${ }^{7}$ and the Strategies Toward Ending Preventable Maternal Mortality. These include targets to reduce stillbirth and maternal and newborn mortality. The United Nations and WHO have also confirmed their commitment to address preventable maternal and neonatal deaths with the launch of the Sustainable Development Goals and the Global Strategy for Women's, Children's and Adolescents' Health (2016-2030). This provides a framework for the implementation, follow-up and review of progress towards relevant targets.

In light of the clustering of morbidity and mortality around the time of childbirth and of the need for practical tools for improvement of the quality of care, WHO have also developed the WHO Safe Childbirth Checklist (SCC). ${ }^{9}{ }^{10}$ This Checklist, built on the success of the WHO Safe Surgical Checklist, ${ }^{11}$ aims to help birth attendants through the process of the childbirth and the management of complications as they arise.

The Checklist is a list of essential evidencebased practices drawn from existing WHO guidelines. These practicesshould be performed at every birth to ensure the best outcome. The 


\section{Key questions}

\section{What is already known about this topic?}

- Provision of life-saving interventions alone is not enough to reduce maternal and neonatal mortality; coverage needs to be matched with improvements in the quality of care.

- WHO Safe Childbirth Checklist (SCC) aims to ensure proven essential maternal and perinatal care practices are performed by skilled birth attendants on admission, just before birth, soon after birth and on discharge.

- Implementing such novel tools requires a carefully deliberated implementation strategy. It is important to test them in a diverse range of settings to help design the appropriate implementation support.

\section{What are the new findings?}

- WHO established the WHO SCC Collaboration to explore questions addressing compliance, barriers to and success factors of effective and sustained use of the Checklist (pilot edition) in a range of settings around the world.

- Most collaboration members established teams to lead the implementation of the Checklist, often with the use of formal training sessions as an initial step to launch the Checklist. This was often followed by ongoing supervision to monitor and encourage Checklist use.

- The WHO SCC was easy to use and was felt to be of high value. End users with more experience, those who had not received any training and those who lacked supervisory support were more uncertain about the use and success of the Checklist.

- A critical step in all settings was the adaptation of the Checklist to reflect local context and national protocols and standards.

\section{Recommendations for policy}

- This work confirms the need for engaging key stakeholders, assessing the baseline situation and establishing a team of people with a wide range of professional backgrounds and roles before formally launching a novel tool such as the WHO SCC.

- It is valuable to assess the initial knowledge and attitude of both supervisors and end users to better target training efforts.

- Local adaptation with ongoing supervision, effective coaching and monitoring and sharing of data will encourage and motivate end users towards sustainable use of the tool.

best practices are organised around four 'pause points', in other words critical moments where the birth attendant should pause to confirm what they are supposed to do or check if they have completed essential birth practices. The four pause points are: on admission, just before birth, soon after birth and on discharge. An early pilot study has shown that it markedly improves the delivery of essential practices by healthcare workers, ${ }^{9}$ thereby contributing to the improvement of quality of care.

More extensive information about the Checklist can be found on WHO website: http://www.who.int/ patientsafety/implementation/checklists/childbirth/en/.

\section{THE WHO SCC COLLABORATION AND EVALUATION}

Previous experience implementing such novel tools revealed the importance of testing them in a diverse range of settings and providing appropriate implementation support. ${ }^{12-14}$ When WHO released the WHO Surgical Safety Checklist, it was evident that success was driven by the presence of a defined implementation strategy. ${ }^{15}$

WHO therefore established a research collaborative- the WHO SCC Collaboration (the Collaboration) - to explore factors influencing use of the Checklist (pilot edition) in a range of settings around the world. ${ }^{16}$ Between November 2012 and March 2015, a total of 34 interested institutions working in over 200 sites in 29 countries across all six WHO regions registered projects with the Collaboration, and agreed to conduct implementation research and share their experiences as their projects progressed. Groups explored a range of questions that addressed compliance, barriers to and success factors of effective and sustained use of the Checklist. Members, as listed in table 1, were involved in frequent webinars and provided regular progress reports throughout the duration of the collaborative.

As part of the Collaboration, a formal evaluation was undertaken by WHO, Ariadne Labs and the Harvard T H Chan School of Public Health, that looked at facilitating factors and barriers to using the Checklist across different settings and helped inform the development of the final version of the WHO SCC and its implementation guide. ${ }^{17}$ Project leads from each collaboration site were asked to distribute two surveys: the first targeted end users experience of using the Checklist, and the second targeted implementation teams-those involved in planning, leading or helping with introduction of the Checklistand their experience of introducing the Checklist at the targeted facility or group of facilities.

Over the 6-week evaluation data collection period, a total of 134 end users and 38 implementation teams responded to the surveys from 39 sites, across 19 countries. Lessons from Evaluation present the key findings from this process.

\section{LESSONS FROM THE EVALUATION Implementation process}

For the most part, collaboration members established formal implementation teams. Over half modified the Checklist before introduction (58\%) with a view to adapting it to their local context. For example, several teams removed HIV and replaced it with a hepatitis B check as this was felt to be more of an issue for their respective populations.

Nearly all teams reported that they had trained end users to use the Checklist (95\%) and several stated that they had also organised question and answer sessions. However, although most end users received education about the purpose of the Checklist prior to its introduction (83\%), fewer than two-thirds stated they were trained to use it $(64 \%)$. Even fewer were coached or supervised while using the Checklist (34\%).

When training was undertaken, topics included introduction on the importance of the Checklist and patient safety and how to use the Checklist, but also technical skills around the areas covered by the Checklist, such as 
Table 1 Collaboration members: location and institution leading the study

\section{WHO region}

African region

Africa (multisite project)

Bahir Dar, Amhara, Ethiopia

Conakry, Guinea

Kayes Region, Mali

Port Harcourt, Rivers State, Nigeria

Orognum, Rivers State, Nigeria

United Republic of Tanzania

Uganda

\section{Region of the Americas}

Buenos Aires, Argentina

Rio Grande do Sul, Brazil

São Paulo, Brazil

Colombia

Mexico

Lima, Peru

Punta del Este, Uruguay

Brazil, Mexico, Peru \& Venezuela (multinational project)

\section{South-East Asia region}

Dhaka, Bangladesh

South-western Bangladesh

Rajasthan, India

Pondicherry, India

Banda Aceh and Yogyakarta, Indonesia

Colombo, Sri Lanka

\section{European region}

Mondovi, Piedmont, Italy

Tuscany, Italy

Barcelona, Spain

\section{Eastern Mediterranean region}

Cairo, Egypt

Mashhad, Islamic Republic of Iran

Zgharta, Lebanon

Kyber Pakhtunkwha, Pakistan

Rawalpindi, Pakistan

Khartoum, Sudan

Khartoum, Sudan

Western Pacific region

Pudong, Shanghai, China

Manila, Philippines

\section{Institution}

Millennium Villages Project, USA

University of Aberdeen, UK

Jhpiego, USA

United States Agency for International Development (USAID) Applying Science to Strengthen and Improve Health Systems

University of Port Harcourt Teaching Hospital

Rivers State Primary Health Care Management Board

African Medical and Research Foundation (AMREF) Health Africa

Management Sciences for Health, USA

\section{Hospital Alemán}

School of Nursing, Universidade Federal do Rio Grande do Sul

Conjunto Hospitalar do Mandaqui

Universidad Nacional de Colombia

The National Commission of Medical Arbitration (CONAMED, Comisión Nacional de Arbitraje Médico)

Hospital National Dos de Mayo

Sanatorio Semm-Mautone

Instituto Nacional de Salud Pública

Centre for Reproductive Health and International Centre for Diarrhoeal

Disease Research

James P Grant School of Public Health

Jhpiego

Pondicherry Institute

University of Göttingen, Germany

De Soysa Hospital for Women

\section{Azienda Sanitaria Locale $\mathrm{Cn} 1$}

Clinical Risk Management and Patient Safety Center

Hospital del Mar-Parc de Salut Mar

Ain Shams Faculty of Medicine

Mashhad University of Medical Sciences

Saydet Zgharta Hospital

University of Göttingen, Germany

Holy Family Hospital, Rawalpindi Medical College

Royal Care International Hospital

Omdurman Maternity Hospital

Nursing School of the Second Military Medical University and Shanghai

Pudong New Area People's Hospital

Medical City Hospital 
hand hygiene or plotting a partograph. Two organisations reported using simulation and one completed 'training of trainers' for obstetricians and the nurse matron.

The implementation teams generally found the Checklist easy or very easy to introduce $(81 \%)$. Most reported that they had adequate support from senior leadership $(84 \%)$ throughout the introduction phase of the Checklist. However, $41 \%$ of teams reported that they had less financial resources than needed while $30 \%$ reported inadequate human resources to support implementation of the Checklist.

When presented with different curves showing how the Checklist might have been taken up over time, the majority of respondents identified with an initial slow then rapid and steady use over time (s-shaped curve B in figure 1).

\section{Implementation results}

End users were willing or extremely willing to use the Checklist when first introduced (79\%). They themselves reported that midwives and nursing staff were the most willing, while obstetricians/gynaecologists were the most resistant.

End users found the Checklist easy to use (33\% very easy, $57 \%$ easy). The proportion who received training were more likely to report the Checklist as easy/very easy to use $(70 \%)$ compared with those who had not received any training $(15 \%)$.

Some $67 \%$ of end users thought that the Checklist improved their practice around the time of childbirth. They commented that the Checklist was helpful in preparation for delivery and in reminding to complete all essential steps, while providing early identification of risks and complications.

"It has enabled us to quickly detect risk... and for the prompt management of complications of mother and newborn." (End user)

Nursing staff and medical doctors were more likely to believe that the Checklist improved practice than
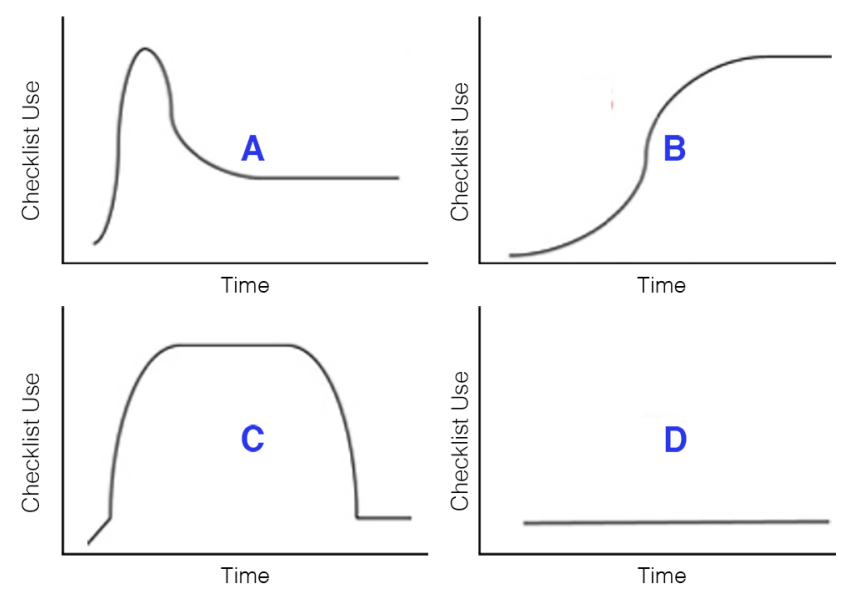

Graph A - 17\% (5) Graph B - 52\% (15)

Graph C-14\% (4) Graph D - 17\% (5)

Figure 1 Uptake of the Checklist over time as perceived by implementation teams. midwives, of whom majority felt it did not. Nearly three quarters of respondents felt the Checklist improved awareness of patient safety, and more than two-thirds of respondents felt that the Checklist improved communication and teamwork in their facility. Once again, midwives were less likely to believe the Checklist improved awareness or communication and teamwork. Respondents with more years of experience, those who had not received any formal training and those who did not have any supervisory support were less likely to believe that the Checklist improved practice, awareness of patient safety or communication and teamwork.

Implementation teams felt that the Checklist was of high value; $62 \%$ felt that significant or very significant progress had been made in the safety of childbirth as a result of using the Checklist. A large majority felt that using the Checklist significantly improved maternal and newborn care $(72 \%)$ and nearly all of the teams said they would want the Checklist to be used for a family member or close friend $(92 \%)$. Some $86 \%$ said that they would like to see the Checklist used in their facility in the future.

"Our use of the checklist highlights deficiencies in certain areas of practice. Obstetric emergencies were generally managed well but hand hygiene required improvement. There were deficiencies in preparation for key steps_partly highlighting a lack of key equipment." (Implementation lead)

"The Checklist is a good idea and is easy to use. The uptake of this pilot study to global use will be a welcome development." (Implementation lead)

\section{Facilitating factors}

End users and implementation team respondents were asked to list three factors that contributed positively to use of the Checklist. Responses included: characteristics of the tool, the way in which it was implemented and strong leadership (table 2). One end user noted that it worked 'to establish a relationship of trust between the provider and patient', while a common theme was well articulated by another end user who stated that the checklist acts as a guide in monitoring and supervising labor hence enhancing patient's care and safety.'

Leadership engagement was considered critical by the implementation teams, most notably in motivating staff to use the Checklist and ensuring adequate supplies. The process of adaptation to reflect local context and ensure buy-in was also noted as useful in engaging leaders. Involvement of patient safety and quality assurance units were also among the top factors that contributed positively to use of the Checklist.

"Leadership engagement at the facility and from obs/gyn who helped with adaptation, training and ongoing supervision"

"Interest of the Obs/Gyn experts of the country who helped us to contextualise and adapt the checklist, and to train the medical doctors who are involved with the implementation of the checklist." (Implementation lead)

Training, the availability of ongoing monitoring and supervision following training, as well as motivation were 
Table 2 Most commonly cited factors that contributed positively to use of the Checklist

\section{End users}

Easy to complete

Good way to prepare for delivery and covers main aspects of management.

Serves as an important reminder to perform essential practices

Helps with early identification and prevention of risk factors Good for communication and sharing information with the team

\section{Implementation team}

Easy to use with good design

Motivation from leadership

Involvement of patient safety and quality assurance units

Training and supervision on Checklist use

Enthusiasm of staff to use Checklist

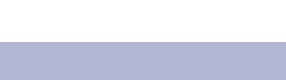

considered key to success. Many sites had senior staff who were trained to mentor, supervise and encourage others to use the Checklist.

Some facilities made the Checklist mandatory which was thought to facilitate implementation.

\section{Challenges to implementation}

Both end users and implementation teams also faced a number of challenges.

Challenges reported by the implementation teams included initial staff resistance, an initial impression of complexity about using the Checklist, the need for local adaptation and lack of supplies (both of the Checklist itself and essential birth supplies).

End users most commonly reported challenges relating to the working environment. Some examples include the inability to use the Checklist when they were too busy or understaffed and lack of awareness of the Checklist by other staff (due to turnover or other unspecified reasons).

However, both groups also reported effective solutions, which they implemented in response to a number of these challenges. These are presented in table 3. Training and ongoing supervision were cited as the main solutions, alongside ensuring the availability of key supplies. Several teams also varied the physical location and form of the Checklist, such as creating wall posters of it.

\section{CONCLUSION}

The experiences of end users and implementation teams using the WHO SCC (pilot edition) across a range of settings from high to low-income countries highlight the need to engage local leadership, enable local ownership and ensure local relevance and acceptability in facilitating initial implementation and successful

Table 3 Challenges to implementation and suggested solutions

Challenge Solutions

Resistance to using the Checklist and a lack of staff Education, ongoing supervision and training specifically addressing motivation knowledge gaps; Educating physicians and their supervisors on the importance and value of the Checklist

Lack of understanding on how to use the Checklist

Educating staff on how to use the Checklist, developing leaflets and other job aids

Perception of increased workload related to Checklist Education on the importance of the Checklist and essential practices use

Limited Checklist supply

Working with management to develop a supply system; Identifying a leader responsible for ensuring supplies

Lack of essential birth supplies

Advising management to request the identified critical drugs/ equipment; Implementers to conduct assessments and arrange provision of missing supplies

Inadequate staff including intermittent attendance

Focus on attendance; Encourage birth attendants to ask birth companions for assistance

Staff turnover Conducting ongoing periodic training sessions

Care of women and newborns at different places in the facility

Splitting Checklist pause points into separate documents for each of the different sites for care

Forgetting to use the Checklist Placing the Checklist in visible places; creating large posters for walls

Insufficient trained birth attendants to manage several Encouraging birth attendants to use birth companions for assistance women presenting in labour at the same time

Inconsistencies with local and national guidelines Adaptation/modification of the Checklist

Lack of clinical skills required by Checklist Arranging trainings for essential birth practices 
uptake. Adaptation of the Checklist is essential to ensure consistency with local guidelines and to prompt willingness among end users to adopt the Checklist. Several studies have highlighted similar key factors to successful implementation. ${ }^{15} 1819$ In addition, obstetricians and non-specialist medical doctors were the most resistant to using the Checklist. However, it was the midwives who felt that the Checklist did not improve practice. Implementation efforts should focus on overcoming reluctance from these professional groups through early engagement and education, for example. ${ }^{20}$

It is also important to ensure training and provide supervision throughout the implementation process. Unsurprisingly, those who received training or were supervised were more likely to believe that the Checklist improved practice, increased awareness of patient safety and/or improved communication and teamwork. This is in line with previous studies ${ }^{15}$ and further supports the role of coaching. ${ }^{21}$ Furthermore, we experienced a variety of challenges in implementing and using the Checklist. End users most commonly cited that they were too busy or understaffed to use the Checklist or that a lack of awareness of the Checklist by other staff had hampered its effective use. Teams also faced challenges with supplies, both with essential birth supplies and the Checklist itself. Implementation teams used an array of solutions to address these challenges, many of which focused on multidisciplinary education and regular training sessions.

The responses from which we have drawn these insight were subjective. It is important to conduct formal external evaluation which should include measurement of compliance with Checklist use, impact on adherence to essential birth practices and ultimately whether or not it improves maternal and neonatal outcomes. Many of the Collaboration members have undertaken research in these areas and have already set about publishing their results. ${ }^{20}$ Ariadne Labs are also currently undertaking a randomised controlled trial in India to explore the effect of Checklist use on maternal and newborn outcomes. ${ }^{21}$ The WHO SCC Collaboration was invaluable in helping us develop the ultimate version of the WHO SCC ${ }^{3}$ and its Implementation Guide. These were formally launched in December 2015 and are available on the WHO website. ${ }^{17}$ The Guide provides further details on the key facilitating factors identified in this evaluation, and highlights important factors for engagement, launch of the Checklist and ongoing support. The implementation guide is targeted for the facility level but it includes advice for a national level approach.

The Checklist is in line with the comprehensive WHO Framework for Improvement of the Quality of Maternal and Newborn Care ${ }^{22}$ that aims to achieve coverage of key practices, people-centred outcomes and eventually improved health outcomes. Eight standards of care have been developed to assist countries translate the framework into practice, and to make it even more explicit and actionable, each standard is accompanied by several quality statements that have been formulated to drive measurable improvements in the quality of care around the time of childbirth. These are valuable tools for policymakers ${ }^{22}$ providing a basis for broader national level quality improvement strategies. As part of the national efforts, WHO particularly encourages low and middle-income countries to implement the WHO SCC in health facilities, using the implementation guide to help inform the implementation process and support the sustained use of the Checklist.

Acknowledgements The authors would like to thank Dr Gulmezoglu and $\mathrm{Mr}$ Banica for their oversight and support of this project, as well as the WHO SCC Collaboration members.

Contributors WRGP and SBN are joint first authors. All authors contributed to the writing and editing of the manuscript. WRGP, SBN and IL were responsible for the conception of the WHO Safe Childbirth Checklist Collaboration, which was subsequently run by SBN and WRGP with oversight of KT, NDK, NR, NK, LRH, KS and MM. All authors contributed to the design of the survey and subsequent analysis. TRD undertook statistical analysis.

Disclaimer The author(s) is(are) staff member(s) of the World Health Organization. The author(s) alone is(are) responsible for the views expressed in this publication and they do not necessarily represent the views, decisions or policies of the World Health Organization.

\section{Competing interests None declared.}

Provenance and peer review Not commissioned; internally peer reviewed. Data sharing statement WHO Safe Childbirth Checklist is available online at Open Access @ C World Health Organization [2017]. Licensee BMJ. This is an open access article distributed under the terms of the Creative Commons Attribution N-Noncommercial IGO License (CC BY 3.0 IGO), which permits use, distribution, and reproduction for non-commercial purposes in any medium, provided the original work is properly cited. In any reproduction of this article there should not be any suggestion that WHO or this article endorse any specific organization or products. The use of the WHO logo is not permitted. This notice should be preserved along with the article's original URL.

(C) Article author(s) (or their employer(s) unless otherwise stated in the text of the article) 2017. All rights reserved. No commercial use is permitted unless otherwise expressly granted.

\section{REFERENCES}

1. UNICEF, World Health Organization. The World Bank, United Nations Population Division, the Interagency Group of Child Mortality Estimation (UN IGME). Levels and trends in child mortality. New York 2015.

2. Lawn JE, Blencowe $\mathrm{H}$, Waiswa $\mathrm{P}$, et al. Stillbirths: rates, risk factors, and acceleration towards 2030. Lancet 2016;387:587-603.

3. World Health Organization. Maternal mortality. World Health Organization 2015 http://www.who.int/mediacentre/factsheets/ fs348/en/ (accessed 10 Dec 2016).

4. Say L, Chou D, Gemmill A, et al. Global causes of maternal death: a WHO systematic analysis. Lancet Glob Health 2014;2:e323-e333.

5. Souza JP, Gülmezoglu AM, Vogel J, et al. Moving beyond essential interventions for reduction of maternal mortality (the WHO Multicountry Survey on Maternal and Newborn Health): a crosssectional study. Lancet 2013;381:1747-55.

6. Barros AJ, Ronsmans C, Axelson $\mathrm{H}$, et al. Equity in maternal, newborn, and child health interventions in countdown to 2015: a retrospective review of survey data from 54 countries. Lancet 2012;379:1225-33.

7. World Health Organization, UNICEF. Every Newborn: an action plan to end preventable deaths:Executive summary. Geneva: World Health Organization, 2014. http://www.who.int/entity/life-course/p artners/global-strategy/globalstrategyreport2016-2030-lowres.pdf? ua $=1$. (accessed 10 Dec 2016).

8. World Health Organization. Strategies toward ending preventable maternal mortality (EPMM). Geneva: World Health Organization 2015 
http://apps.who.int/iris/bitstream/10665/153544/1/9789241508483 eng.pdf?ua=1 (accessed Dec 10 2016).

9. Spector JM, Agrawal P, Kodkany B, et al. Improving quality of care for maternal and newborn health: prospective pilot study of the WHO Safe Childbirth Checklist program. PLoS One 2012; 7:e35151-1.

10. Spector JM, Lashoher A, Agrawal P, et al. Designing the WHO Safe Childbirth Checklist program to improve quality of care at childbirth. Int J Gynaecol Obstet 2013;122:164-8.

11. Haynes AB, Weiser TG, Berry WR, et al. A surgical safety checklist to reduce morbidity and mortality in a global population. $N$ Engl J Med 2009;360:491-9.

12. Allegranzi B, Conway L, Larson $\mathrm{E}$, et al. Status of the implementation of the World Health Organization multimodal hand hygiene strategy in United States of America health care facilities. Am J Infect Control 2014;42:224-30.

13. Allegranzi B, Gayet-Ageron A, Damani N, et al. Global implementation of WHO's multimodal strategy for improvement of hand hygiene: a quasi-experimental study. Lancet Infect Dis 2013;13:843-51.

14. Palomar M, Álvarez-Lerma F, Riera A, et al. Impact of a national multimodal intervention to prevent catheter-related bloodstream infection in the ICU: the spanish experience. Crit Care Med 2013;41:2364-72.

15. Perry WRG, Kelley ET. Checklists, global health and surgery: a fiveyear checkup of the WHO Surgical Safety checklist programme. Clin Risk 2014;20:59-63.
16. World Health Organization. The WHO Safe Childbirth Checklist Collaboration. http://www.who.int/patientsafety/implementation/ checklists/childbirth_collaboration_engaging/en/ (Accessed 10 December 2016).

17. World Health Organization. WHO Safe Childbirth Checklist Implementation Guide Geneva: WHO Press; 2015. http://www. who.int/patientsafety/implementation/checklists/childbirthchecklist_implementation-guide/en/ (Accessed 10 December 2016)

18. Treadwell JR, Lucas S, Tsou AY. Surgical checklists: a systematic review of impacts and implementation. BMJ Qual Saf 2014;23.

19. Thomassen $\varnothing$, Espeland A, Søfteland E, et al. Implementation of checklists in health care; learning from high-reliability organisations. Scand J Trauma Resusc Emerg Med 2011;19:53.

20. Patabendige M, Senanayake H. Implementation of the WHO safe childbirth checklist program at a tertiary care setting in Sri Lanka: a developing country experience. BMC Pregnancy Childbirth $2015 ; 15: 12$

21. Hirschhorn LR, Semrau K, Kodkany B, et al. Learning before leaping: integration of an adaptive study design process prior to initiation of BetterBirth, a large-scale randomized controlled trial in Uttar Pradesh, India. Implement Sci 2015;10:117.

22. World Health Organization. Standards for improving quality of maternal and newborn care in health facilities. Geneva: World Health Organization http://apps.who.int/iris/bitstream/10665/ 249155/1/9789241511216-eng.pdf?ua=1 (Accessed 10 December 2016). 